\title{
Physics and Results from the AMANDA-II High Energy Neutrino Telescope
}

Steven W. Barwick, for the AMANDA Collaboration

J. Ahrens ${ }^{11}$, X. Bai ${ }^{1}$, S.W. Barwick ${ }^{10}$, T. Becka ${ }^{11}$, K.-H. Becker ${ }^{2}$, E. Bernardini ${ }^{4}$, D. Bertrand ${ }^{3}$, F. Binon ${ }^{3}$, A. Biron ${ }^{4}$, S. Böser ${ }^{4}$, O. Botner $^{16}$, O. Bouhali ${ }^{3}$, T. Burgess ${ }^{17}$, S. Carius $^{6}$, T. Castermans ${ }^{12}$, D. Chirkin ${ }^{9,2}$, J. Conrad ${ }^{16}$, J. Cooley ${ }^{14}$, D.F. Cowen ${ }^{8}$, A. Davour ${ }^{16}$, C. De Clercq $^{18}$, T. DeYoung ${ }^{14,19}$, P. Desiati ${ }^{14}$, J.-P. Dewulf ${ }^{3}$, P. Doksus ${ }^{14}$, P. Ekström ${ }^{17}$, T. Feser ${ }^{11}$, T.K. Gaisser ${ }^{1}$, R. Ganupati ${ }^{14}$, M. Gaug ${ }^{4}$, H. Geenen ${ }^{2}$, L. Gerhardt ${ }^{10}$, A. Goldschmidt ${ }^{7}$, A. Hallgren ${ }^{16}$, F. Halzen ${ }^{14}$, K. Hanson ${ }^{14}$, R. Hardtke ${ }^{14}$, T. Hauschildt ${ }^{4}$, M. Hellwig ${ }^{11}$, P. Herquet ${ }^{12}$, G.C. Hill ${ }^{14}$, P.O. Hulth ${ }^{17}$, K. Hultqvist ${ }^{17}$, S. Hundertmark ${ }^{17}$, J. Jacobsen ${ }^{7}$, A. Karle ${ }^{14}$, L. Köpke ${ }^{11}$, M. Kowalski ${ }^{4}$, K. Kuehn ${ }^{10}$, J.I. Lamoureux ${ }^{7}$, H. Leich ${ }^{4}$, M. Leuthold ${ }^{4}$, P. Lindahl ${ }^{6}$, J. Madsen ${ }^{15}$, K. Mandli, P. Marciniewski ${ }^{16}$, H.S. Matis ${ }^{7}$, C.P. McParland ${ }^{7}$, T. Messarius ${ }^{2}$, Y. Minaeva ${ }^{17}$, P. Miočinović ${ }^{9}$, R. Morse ${ }^{14}$, R. Nahnhauer ${ }^{4}$, T. Neunhöffer ${ }^{11}$, P. Niessen ${ }^{18}$, D.R. Nygren ${ }^{7}$, H. Ogelman ${ }^{14}$, Ph. Olbrechts ${ }^{18}$, C. Pérez de los $\operatorname{Heros}^{16}$, A.C. Pohl ${ }^{6}$, P.B. Price ${ }^{9}$, G.T. Przybylski ${ }^{7}$, K. Rawlins ${ }^{14}$, E. Resconi ${ }^{4}$, W. Rhode ${ }^{2}$, M. Ribordy ${ }^{4}$, S. Richter ${ }^{14}$, J. Rodríguez Martino ${ }^{17}$, D. Ross ${ }^{10}$, H.-G. Sander ${ }^{11}$, K. Schinarakis ${ }^{2}$, T. Schmidt ${ }^{4}$, D. Schneider ${ }^{14}$, R. Schwarz ${ }^{14}$, A. Silvestri ${ }^{10}$, M. Solarz ${ }^{9}$ G.M. Spiczak ${ }^{15}$, C. Spiering ${ }^{4}$, D. Steele ${ }^{14}$, P. Steffen ${ }^{4}$, R.G. Stokstad ${ }^{7}$, P. Sudhoff ${ }^{4}$, K.-H. Sulanke ${ }^{4}$, I. Taboada ${ }^{13}$, L. Thollander ${ }^{17}$, S. Tilav ${ }^{1}$, W. Wagner ${ }^{2}$, C. Walck ${ }^{17}$, C.H. Wiebusch ${ }^{4,20}$, C. Wiedemann ${ }^{17}$, R. Wischnewski ${ }^{4}$, H. Wissing ${ }^{4}$, K. Woschnagg ${ }^{9}$, G. Yodh ${ }^{10}, \mathrm{~S}$. Young ${ }^{10}$

(1) Bartol Research Institute, University of Delaware, Newark, DE 19716, USA

(2) Fachbereich 8 Physik, BUGH Wuppertal, D-42097 Wuppertal, Germany

(3) Université Libre de Bruxelles, Science Faculty CP230, Boulevard du Triomphe, B-1050 Brussels, Belgium

(4) DESY-Zeuthen, D-15735 Zeuthen, Germany

(6) Dept. of Technology, Kalmar University, S-39182 Kalmar, Sweden

(7) Lawrence Berkeley National Laboratory, Berkeley, CA 94720, USA

(8) Dept. of Physics, Pennsylvania State University, University Park, PA 16802, USA

(9) Dept. of Physics, University of California, Berkeley, CA 94720, USA

(10) Dept. of Physics and Astronomy, University of California, Irvine, CA 92697, USA

(11) Institute of Physics, University of Mainz, Staudinger Weg 7, D-55099 Mainz, Germany

(12) University of Mons-Hainaut, 7000 Mons, Belgium

(13) Departamento de Física, Universidad Simón Bolívar, Apdo. Postal 89000, Caracas, Venezuela

(14) Dept. of Physics, University of Wisconsin, Madison, WI 53706, USA

(15) Physics Dept., University of Wisconsin, River Falls, WI 54022, USA

(16) Division of High Energy Physics, Uppsala University, S-75121 Uppsala, Sweden

(17) Dept. of Physics, Stockholm University, SCFAB, SE-10691 Stockholm, Sweden

(18) Vrije Universiteit Brussel, Dienst ELEM, B-1050 Brussel, Belgium

(19) Present address: Santa Cruz Institute for Particle Physics, University of California, Santa 
Cruz, CA 95064, USA

(20) Present address: CERN, CH-1211, Genève 23, Switzerland

\begin{abstract}
This paper briefly describes the principle of operation and science goals of the AMANDA high energy neutrino telescope located at the South Pole, Antarctica. Results from an earlier phase of the telescope, called AMANDA-B10, demonstrate both reliable operation and the broad astrophysical reach of this device, which includes searches for a variety of sources of ultrahigh energy neutrinos: generic point sources, Gamma-Ray Bursts and diffuse sources. The predicted sensitivity and angular resolution of the telescope were confirmed by studies of atmospheric muon and neutrino backgrounds. We also report on the status of the analysis from AMANDA-II, a larger version with far greater capabilities. At this stage of analysis, details of the ice properties and other systematic uncertainties of the AMANDA-II telescope are under study, but we have made progress toward critical science objectives. In particular, we present the first preliminary flux limits from AMANDA-II on the search for continuous emission from astrophysical point sources, and report on the search for correlated neutrino emission from Gamma Ray Bursts detected by BATSE before decommissioning in May 2000. During the next two years, we expect to exploit the full potential of AMANDA-II with the installation of a new data acquisition system that records full waveforms from the in-ice optical sensors.
\end{abstract}

\title{
1. INTRODUCTION
}

Nature provides precious few information carriers from the deep recesses of space, and it is imperative to develop techniques to exploit them all. Throughout history, the photon messenger has made vital contributions to the understanding of the observable Universe. In this paper, we present results from a new generation of telescope designed to detect a very different kind of information carrier, high energy neutrinos (where $E_{\nu}>1 \mathrm{TeV}$ ). The search for astronomical sources of high energy neutrinos is one of the central missions of the Antarctic Muon and Neutrino Detector Array (AMANDA), located close to the geographic South Pole in Antarctica (Andres et al. 2000). It is possible to identify sources (i.e, astronomy) with neutrinos because they are neutral and stable. The most powerful detection technique relies on the observation of a muon that is created by charged-current interactions between a neutrino and atomic nuclei in and around the instrumented volume of the detector. At the energies of interest, the direction of the muon is highly correlated with the direction of the parent neutrino. The correlation is usually characterized by the mean angle of deviation, $\theta_{\nu \mu} \sim 0.65^{\circ} /\left(E_{\nu}\right)^{0.48}$, where $E_{\nu}$ is the neutrino energy in units of $10^{12} \mathrm{eV}$. The correlation between the detected muon and neutrino direction is helped by the fact that the higher energy muons are more readily detected because they 
emit more Cherenkov photons and they travel further than lower energy ones. Ideally, the muon angular resolution of the telescope should be comparable to or less than the angular correlation between muon and neutrino. The muon energy can be estimated from the relative increase in Cherenkov emission mainly due to pair production and bremsstrahlung.

Ultrahigh energy (UHE) neutrinos with energies in the $\mathrm{TeV}$ range and higher may be produced by a variety of sources. Particle physics exotica like WIMPs and topological defects are expected to produce neutrinos in their annihilation or decay (Bertin, Negri, \& Orloff 2002), and models of astrophysical phenomena such as gamma-ray bursts, active galactic nuclei, supernovae and microquasars (Gaisser et al. 1995; Levinson and Waxman 2002; Dermer and Atoyan 2001) also predict UHE neutrino fluxes. Micro-black holes may be produced in the ice by collisions with extremely high energy neutrinos (Ringwald\&Tu 2002).

AMANDA is sensitive to UHE neutrinos produced by these sources and can provide some of the most stringent tests to date of UHE neutrino production models. More generally, AMANDA and other similar neutrino telescopes open a largely unexplored window on the universe in a region of the energy spectrum bounded between roughly $10^{12} \mathrm{eV}$ and $10^{20} \mathrm{eV}$. In the somewhat narrower energy range between roughly $10^{14} \mathrm{eV}$ and $10^{19} \mathrm{eV}$, photons are absorbed by intervening matter and starlight, and cosmic-ray protons are insufficiently energetic to reach us without experiencing unknown amounts of curvature in intervening magnetic fields, leaving neutrinos as the only known particles that can serve as astronomical messengers. Neutrino telescopes are also sensitive to supernova neutrino bursts at neutrino energies of roughly $10^{7} \mathrm{eV}$.

While the scientific potential of neutrino astronomy is broad, far reaching, and exciting, it is important to keep expectations realistic by assessing the scientific and technical capabilities of current and future neutrino telescopes. In the next section(s), we describe the detection technique, signatures, effective size, and backgrounds that define the capabilities of AMANDA-II.

\section{THE AMANDA DETECTOR}

The essential characteristics of a neutrino telescope have been known for more than two decades and most important features were discussed and specified during a series of workshops devoted to developing the DUMAND concept. In fact, more than four decades ago, Markov suggested that the ocean would be a suitable site for constructing a large neutrino detector based on the detection of Cherenkov light. Halzen and Learned (for brief history of development of high energy neutrino detectors, see Barwick (2000)) introduced a twist on the general scheme by promoting polar ice as suitable medium. Until recently, workable implementations of these sensible ideas have been thwarted by unusual technical and logistical challenges associated with the remote deployment of hardware in media that differ from ordinary purified water in several important details.

The AMANDA-B10 high energy neutrino detector consists of lattice of 302 optical modules (OMs) on 10 strings. Each OM is comprised of a photomultiplier tube (PMT) with passive electronics housed in a glass pressure vessel. The OMs are deployed within a cylindrical volume about $120 \mathrm{~m}$ in diameter and $500 \mathrm{~m}$ 


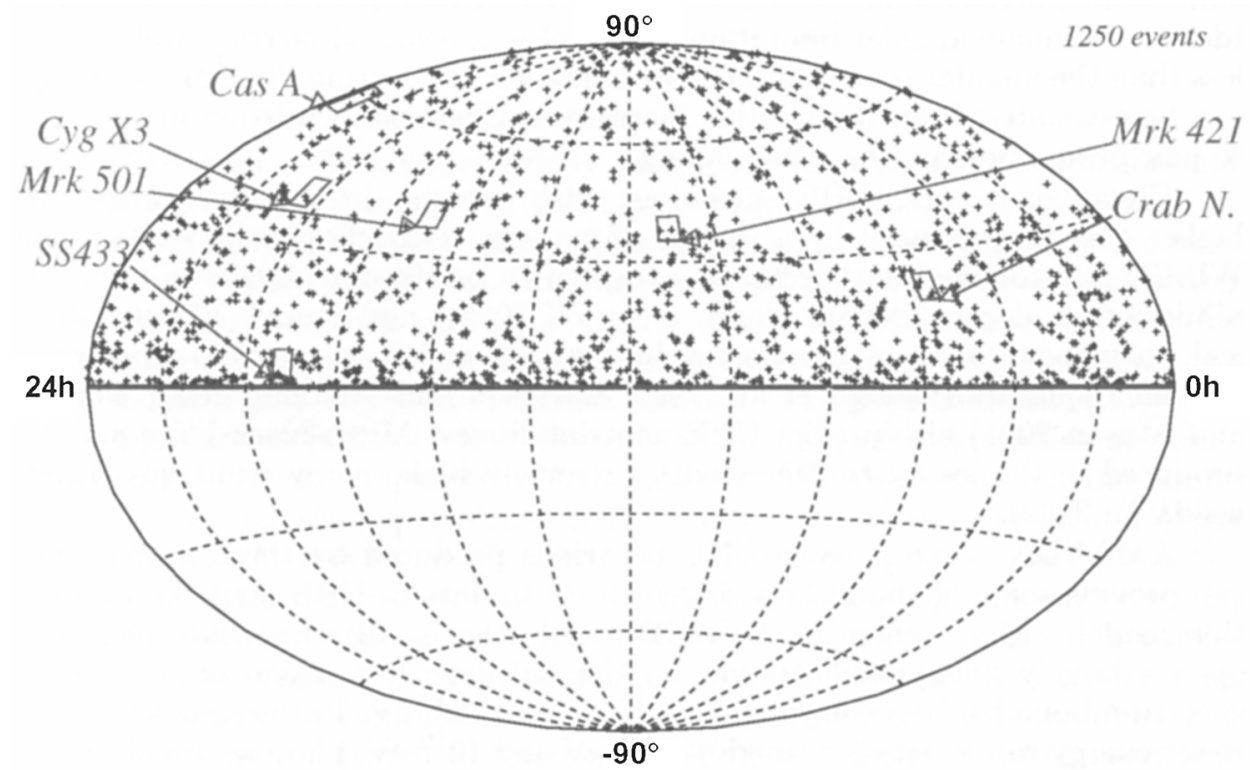

Figure 1. Sky plot obtained from the AMANDA-II point source analysis. Horizontal coordinates are right ascension and vertical coordinates are declination. Also shown are the sky coordinates and characteristic search bin for several potential neutrino sources.

in height at depths between roughly 1500 and $2000 \mathrm{~m}$ below the surface of the South Pole ice cap. In this region the optical properties of the ice are well suited for reconstructing the Cherenkov light pattern emitted by relativistic charged particles (Price, Woschnagg,\&Chirkin 2000). This light is used to reconstruct individual events. An electrical cable provides high voltage to the PMTs and transmits their signal pulses to the surface electronics. A light diffuser ball connected via fiber optic cable to a laser on the surface is used for calibration purposes. Copious down-going cosmic ray muons are also used for calibration purposes.

In January 2000, AMANDA-B10 was enlarged to a total of 19 strings with $667 \mathrm{OMs}$ to form AMANDA-II. This new detector is $200 \mathrm{~m}$ in diameter and approximately the same height and depth as AMANDA-B10.

Planning, design, and construction of the new drill has begun on IceCube, a kilometer-scale device with $4800 \mathrm{OMs}$ on 80 strings (Karle et al. 2002).

The optical sensors respond to the UV-blue dominated cherenkov radiation emitted by neutrino-induced muons or neutrino-induced hadronic and electromagnetic cascades. AMANDA technologies and system architecture were developed, and now proven by five years of operation, to be reliable, durable, and robust. AMANDA was shown to be a functioning neutrino detector by virtue of its ability to reconstruct upward-going muons induced by atmospheric muon neutrinos (Andres et al. 2001; Ahrens et al. 2002a) and detailed comparison 
between experimental data and background simulations. It was shown to be a functioning telescope by comparing the expected pointing accuracy and angular resolution with data obtained from air shower events that simultaneously trigger the SPASE air shower array and AMANDA. The technical capabilities of the embedded hardware were sufficient to accomplish to the primary science missions.

AMANDA-B10 data has also been used to set competitive limits on WIMPs (Ahrens et al. 2002b), monopoles (Niessen\&Spiering 2001), extremely energetic neutrinos (Hundertmark 2001), UHE $\nu_{\mu}$ point sources (Ahrens et al. 2003a) and diffuse fluxes (Ahrens et al. 2003b). The detector is also sensitive to bursts of low energy neutrinos from supernovae (Ahrens et al. 2001). In the following sections, we highlight some of the previously reported work, and discuss new analysis of AMANDA-II data.

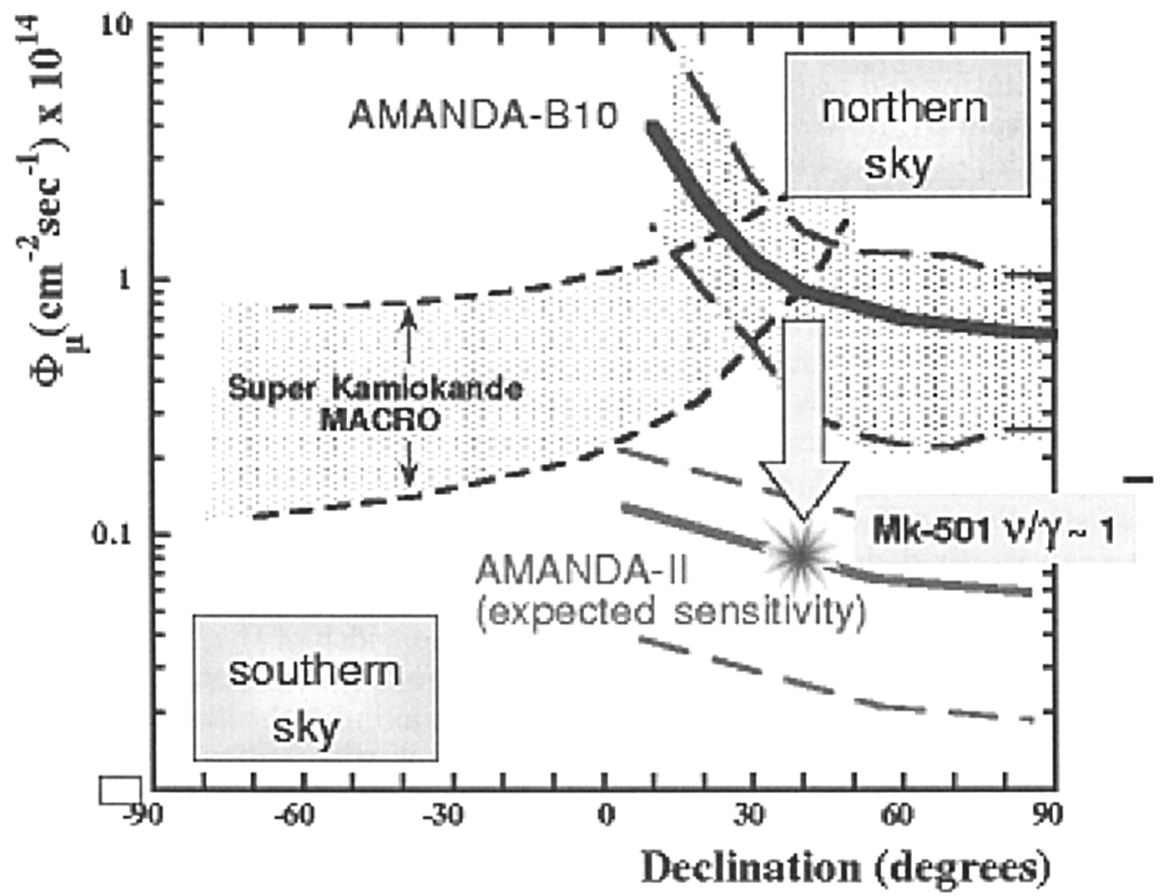

Figure 2. Comparison of current flux limits from AMANDA-B10, SuperKamiokande (Matsuno 2001), and MACRO (Perrone 2001), and projected flux sensitivity for 600 live-days of AMANDA-II. For reference, we indicate the neutrino flux from Markarian 501 assuming the neutrino flux and energy spectrum is identical to the observed highstate gamma ray flux. 


\section{SEARCH FOR UHE $\nu_{\mu}$ FROM POINT SOURCES WITH AMANDA-II}

We have conducted a general search for continuous emission of muon neutrinos from a spatially localized direction in the northern sky. Backgrounds are reduced by requiring a statistically significant enhancement in the number of reconstructed upward-going muons within a small bin in solid angle. The bin size is determined from the predicted angular resolution for muon events. Furthermore, the background for a particular bin can be calculated from the data by averaging over the data external to that bin in the same declination band. In contrast to other specialized analyses used by the AMANDA collaboration, this search is more tolerant of the presence of background, so the signal is optimized on $S / \sqrt{B}$, where $S$ represents the signal and $B$ the background, rather than on $S / B$, which emphasizes signal purity.

Data acquired by AMANDA-B10 in 1997 has been analyzed and the results presented (Ahrens et al. 2003a). That paper details several checks of the simulation programs, such as using SPASE-AMANDA coincidence data to check the absolute pointing and angular resolution, and comparing background predictions with data from trigger to the final selection criteria. Systematic uncertainties in simulation input parameters were studied and included in the final results.

With AMANDA-II data taken in 2000, we gain improved sensitivity over the entire visible sky, and most critically, to events near the horizon since the detector has double the number of PMTs and a larger lever arm in the horizontal dimension. This is illustrated by the 1250 events in Fig. 1. With the exception of the horizon, the event distribution is uniform in declination. In order to achieve blindness in this analysis the right ascension of each event (i.e., its azimuthal angle) was scrambled (at the South Pole this effectively scrambles the event time) before the analysis was finalized. The goal was to search for a statistically significant excess of events from a specific direction in the sky. The analysis divided the sky into non-overlapping $6^{\circ} \times 6^{\circ}$ angular bins at the horizon, and varied the azimuthal width of the bin at larger declinations to maintain approximately constant solid angle. The width of the bin is roughly a factor of 3 larger than the angular resolution of the detector. Four distinct maps were created by shifting the center of the bin to maximize the counts for sources that lie near the edge. Our results are consistent with statistical fluctuation of the background events that remain in the sample. Assuming a customary source spectrum proportional to $E^{-2}$, preliminary flux limits for several sources are presented in Table 1.

In addition to the preliminary limits extracted from data collected in 2000, Table 1 presents projected sensitivities of AMANDA-II using all the data currently on tape. The sensitivity is defined as the predicted average $90 \%$ C.L. limit from an ensemble of experiments with no signal, and is calculated using background levels predicted from off-source data.

Figure 2 summarizes the published experimental muon flux limits as a function of declination. Bands indicate bin-to-bin variation at a given declination due to statistical fluctuation of background events. As seen from Fig. 2, the sensitivity provided by AMANDA-II is sufficient to test the straightforward hypothesis that the brightest $\mathrm{TeV}$ gamma-ray sources emit neutrinos with the same flux 
and energy spectrum proportional to $\mathrm{E}^{-2}$. One consequence of this hypothesis is that the observed ratio of neutrinos to gamma-rays may be larger than unity due to photon absorption within the source, or absorption enroute, but cannot be significantly less than unity because the neutrino cross-section is negligible compared to the photon cross-section. We highlight Markarian 501 in Fig. 2, which shows the neutrino flux limit from B10 along with the projected sensitivity of AMANDA-II with data on tape. If current generation neutrino telescopes do not detect neutrinos from these sources, then the neutrino flux can be can be adjusted through model-specific proton and electron efficiency factors to make models compatible with observational data. Since model assumptions span a large range, it is possible that neutrino telescopes with an order of magnitude improvement in sensitivity will observe a positive signal.

Table 1. Preliminary limits for selected point sources from 2000 data and estimated sensitivities (in parenthesis) of AMANDA-II for data now available on tape ( $\sim 600$ live-days). The sensitivity is defined as the predicted average limit from an ensemble of experiments with no signal, and is calculated using background levels predicted from offsource data. No systematic uncertainties are included.

\begin{tabular}{llll}
\hline Source & Declination & $\mu\left(\times 10^{-15} \mathrm{~cm}^{-2} \mathrm{~s}^{-1}\right)$ & $\nu\left(\times 10^{-8} \mathrm{~cm}^{-2} \mathrm{~s}^{-1}\right)$ \\
\hline SS433 & 5.0 & $6.6(3.1)$ & $4.2(2.0)$ \\
Crab & 22.0 & $6.9(1.5)$ & $7.0(1.6)$ \\
Markarian 421 & 38.2 & $2.4(0.8)$ & $2.6(0.9)$ \\
Markarian 501 & 39.8 & $1.9(0.8)$ & $2.2(0.9)$ \\
Cygnus X-3 & 41.5 & $2.8(0.8)$ & $3.3(0.9)$ \\
Cass. A & 58.8 & $2.2(0.7)$ & $3.2(1.0)$ \\
\hline
\end{tabular}

The point source limits and sensitivities are calculated assuming approximately continuous emission over time. However, many sources exhibit strong temporal variability in the electromagnetic bands. If neutrino emission is strongly correlated with X-ray or gamma-ray variability (Dermer\&Atoyan 2001; Levinson\&Waxman 2002) and the variability is well measured by orbiting or terrestrial telescopes, then sensitivity for a given source should improve with decreasing duration of the flaring episode. The degree of improvement is still under study.

\section{ATMOSPHERIC NEUTRINOS WITH AMANDA-II}

Without a known astronomical source of high energy neutrinos, detector calibration and consistency checks of the predicted response must be carried out by alternative methods. . Atmospheric neutrinos, being the only guaranteed source of high energy signal, provide an important tool to assess neutrino sensitivity. A fraction of the atmospheric muon-neutrinos produced in the northern hemisphere by collisions with cosmic rays travel through the earth, interact with the underlying earth or the ice near AMANDA, and produce a muon which can be detected and reconstructed. Using data collected by AMANDA-B10 in 1997, we reconstructed roughly 300 upward-going muons which, as shown in Fig. 3, are in agreement with the predicted angular distribution. This analysis focuses 

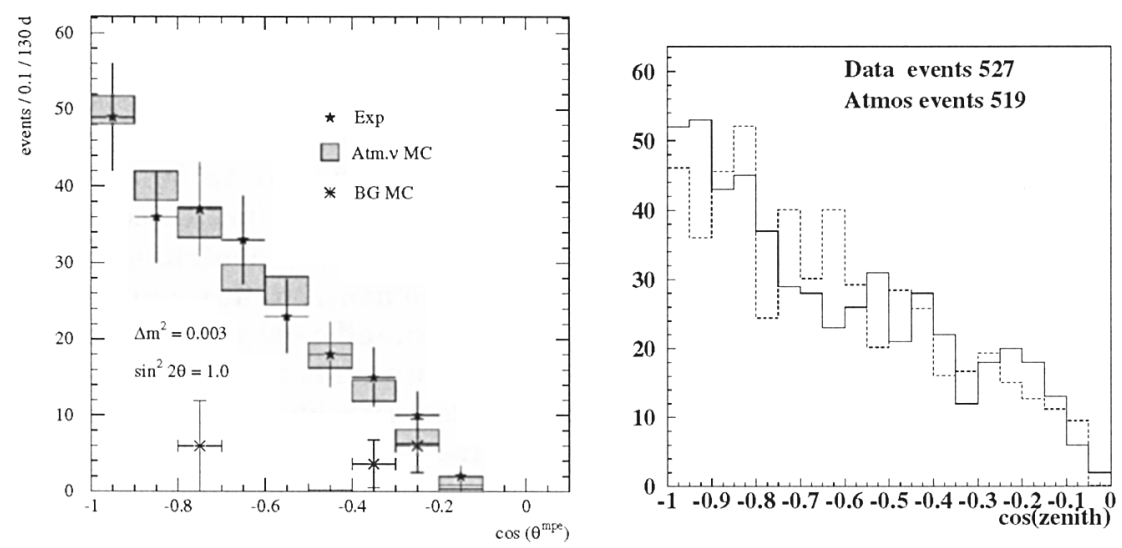

Figure 3. Left: Number of upward-going muon events in AMANDA$\mathrm{B} 10$ data from the year 1997, as a function of zenith angle $(\cos \theta=-1.0$ is vertically up in the detector). The data are shown as dots and the Monte Carlo as boxes. The simulation was performed with the neutrino oscillation parameters as indicated. The predicted signal efficiency is roughly $4 \%$ and the background level is roughly $10 \%$, with both numbers improving near the vertical and degrading near the horizon. Simulations indicate that these events have an energy range given roughly by $60 \mathrm{GeV}<E_{\nu}<300 \mathrm{GeV}$.

Figure 4. Right: Number of upward-going muon events in AMANDA-II data from the year 2000 as a function of zenith angle, using a preliminary set of selection criteria. There are a total of 527 events in the data (solid line), and 519 events predicted by the atmospheric neutrino Monte Carlo (dashed line). Simulations indicate that these events have an energy range given roughly by $100 \mathrm{GeV}<E_{\nu}<$ $1 \mathrm{TeV}$. With more sophisticated selection criteria we expect improved response near the horizon.

on obtaining a pure sample of low energy neutrinos to simplify comparison with simulation. Since this analysis typically produces inefficiencies near the horizon, it is inappropriate to use for sources with harder energy spectra (typically, power law spectra proportional to $E^{-2}$ is targeted).

A preliminary analysis of atmospheric neutrino data taken with AMANDAII demonstrates a substantial increase in the capability of the enlarged detector. Compared to the analysis using AMANDA-B10 data, fewer selection criteria are required to extract a larger and qualitatively cleaner sample of atmospheric neutrino-induced muons. Figure 4 shows the excellent shape agreement between data and simulation achieved with a preliminary set of selection criteria applied. With more sophisticated selection criteria we expect to see roughly twice the number of events shown in the figure (corresponding to 2-3 times more events in AMANDA-II relative to AMANDA-B10 for equivalent live-times) and we also anticipate improved angular response close to the horizon. 

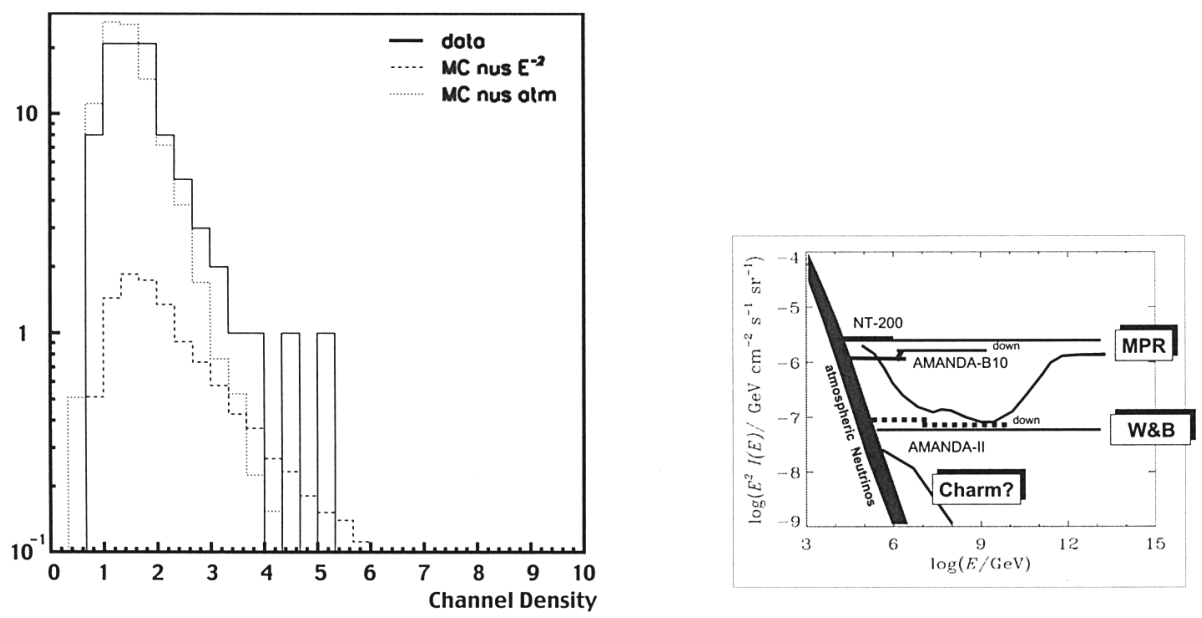

Figure 5. Left: Distributions of the channel density $\rho_{\text {ch }}$ for data, simulated signal and simulated background. The simulated signal assumes a customary $E^{-2}$ power law spectrum at the source and a neutrino flux of $10^{-6} \mathrm{GeV} \mathrm{cm}^{-2} \mathrm{~s}^{-1} \mathrm{sr}^{-1}$. Events are kept if they satisfy $\rho_{\text {ch }}>3$.

Figure 6. Right: Experimental limit (Hill \& Leuthold 2001), projected sensitivities, and theoretical upper bounds (Mannheim, Protheroe, \& Rachen 2000; Waxman \& Bahcall 1999)on the flux of high energy neutrinos from diffusely distributed sources. With the exception of NT-200 at Lake Baikal(Balkanov 2001), all limits are derived for muon neutrinos. The preliminary lower energy AMANDA limits are derived from upward-traveling muons and the extreme high energy (EHE) limits are described by Hundertmark (2001). The horizontal length of the integral limits indicate the energy interval that contains $\sim 90 \%$ of the events, assuming an energy spectrum proportional to $E^{-2}$. The broken curve of high energy background (labeled "Charm?") extending from the atmospheric neutrino curve estimates the additional contribution from prompt muons and neutrinos due to charm decay. 

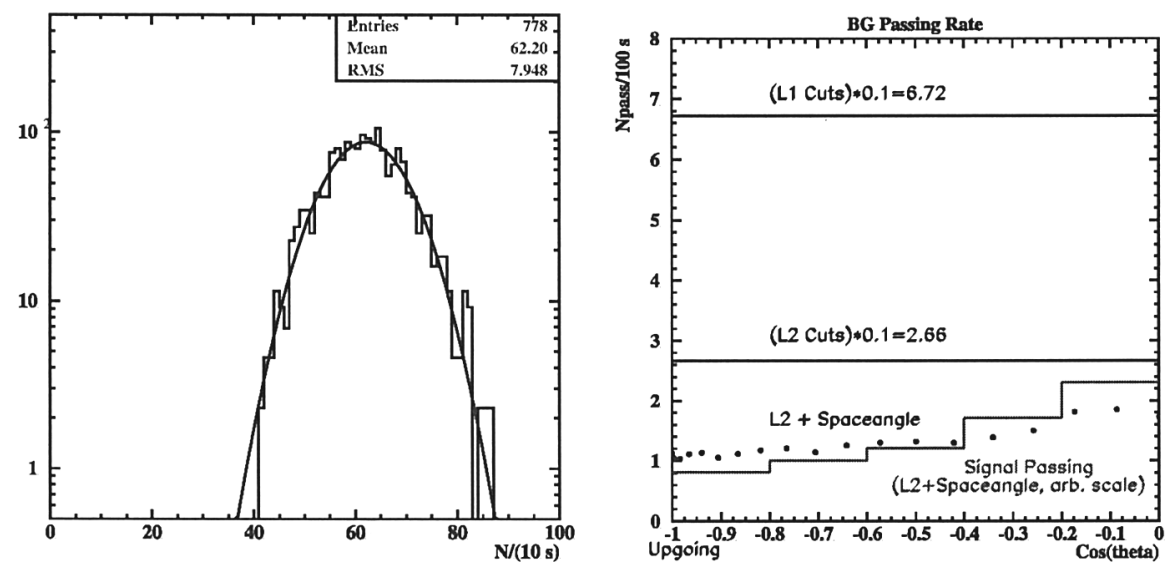

Figure 7. Left: The event count per $10 \mathrm{~s}$ period, demonstrating the good stability and gaussian fluctuation of the AMANDA-II off-time event rate. Some selection criteria have been applied.

Figure 8. Right: Signal and background counts for the GRB perburst analysis as a function of declination. Background counts for various stages of the analysis ( $\mathrm{L} 1=$ level $1, \mathrm{~L} 2=$ level 2 , etc.) are determined for a time interval of 100 seconds, while the signal from the final stage of the analysis (L2+spaceangle)is arbitrarily scaled.

\section{SEARCH FOR $\nu_{\mu}$ FROM DIFFUSE SOURCES WITH AMANDA- II}

The search for diffuse sources of UHE $\nu_{\mu}$-induced muons is similar to the analysis used to detect atmospheric $\nu_{\mu}$-induced muons, as both analyses require a sample of events with low contamination from misreconstructed downward-going atmospheric muons. Since high-energy muons will deposit more energy in the detector volume than low-energy muons, the diffuse analysis further requires that events have a high channel density, $\rho_{\mathrm{ch}}>3$, where the channel density is defined as the number of hit channels per $10 \mathrm{~m}$ tracklength. The background in the signal region is estimated by extrapolating from lower-energy data satisfying $\rho_{\text {ch }}<3$.

Using a $20 \%$ subsample of the AMANDA-II data from 2000, we detect 6 events satisfying all selection criteria. Simulations indicate that we would detect 3.0 events from a UHE neutrino flux at the current best limit (Hill\&Leuthold 2001 ), assuming a customary $E^{-2}$ power law spectrum at the source, and 1.9 events from atmospheric neutrino interactions. (N.B. We use a subsample of the data in order to achieve blindness in this analysis.) The distributions of $\rho_{\mathrm{ch}}$ for data, simulated signal and simulated background are shown in Fig. 5.

The predicted average limit from an ensemble of experiments with no signal, or sensitivity, is roughly $1.3 \times 10^{-6} \mathrm{GeV} \mathrm{cm}^{-2} \mathrm{~s}^{-1} \mathrm{sr}^{-1}$, and the preliminary limit is less than roughly $10^{-6} \mathrm{GeV} \mathrm{cm}^{-2} \mathrm{~s}^{-1} \mathrm{sr}^{-1}$. This is about the same as the limit obtained with the full sample of AMANDA-B10 data from 1997. 
The discovery potential of AMANDA-II (and other current generation neutrino telescopes) is especially high for diffuse signals. The projected sensitivity of AMANDA-II in Fig. ?? indicates that much of the available parameter space is probed. The space is bounded at low energies by the diffuse atmospheric neutrino and muon backgrounds (Lipari 1993). The background floor on this figure depends by the rather uncertain prompt muon and neutrino flux from charm production in the atmosphere. At higher energies, neutrino absorption by the earth implies that diffuse signals originate predominantly from slightly above the horizon. The AMANDA telescope can survey the extremely high energy (EHE) region of the neutrino energy spectrum using techniques that extract bright horizontal events from the less energetic atmospheric background (Hundertmark 2001). AMANDA can provide critical input to more sensitive techniques based on radio Cherekov (Gorham et al. 2002), fluorescence (Scarsi et al. 2001), air shower arrays (Letessier-Selvon et al. 2001), and possibly acoustic (Spiering 2002) signatures from the cascades initiated by such highly energetic interactions. At this early stage of diffuse EHE analysis, the projected sensitivity contains substantial (perhaps factor of 2) uncertainty but AMANDA-II is poised to probe model predictions that lie above the MPR bound(Mannheim, Protheroe, \&Rachen 2000) and those in the vicinity of the W\&B bound(Waxman\&Bahcall 1999). The sensitivity may be better than shown once the analysis techniques incorporate the waveform information that will become available during the next few years.

\section{SEARCH FOR $\nu_{\mu}$ FROM GRBs WITH AMANDA-B10 and -II}

The search for UHE $\nu_{\mu}$-induced muons from gamma-ray bursts (GRBs) utilizes temporal and directional information from satellite-based observations of GRB photons to obtain very large effective area (the per-burst AMANDA-II analysis typically reaches $50 \%$ of the maximum area determined by the hardware trigger conditions). Since AMANDA archives all of the data it collects, the search for correlated high energy neutrino emission uses the archival GRB database from BATSE. The analysis reported here assumes that the neutrino emission occurs over the same time interval that contains $90 \%$ of the gamma-ray photons in the GRB $\left(\mathrm{T}_{90}\right)$. We expand the window by a few seconds around $\mathrm{T}_{90}$ to account for possible early emission as predicted by some models and to reduce the finetuning on the short burst population. The relatively large detection area is a consequence of the modest background rejection, which requires approximately $10^{-4}$ for per-burst analysis and $\sim 2 \times 10^{-5}$ for composite searches. This is contrasted with the approximately $10^{-6}$ rejection requirement for point source analysis and $\sim 10^{-8}$ for the diffuse source analysis. Assuming the predicted spectrum (Waxman\&Bahcall 1997), we search for muon neutrinos in the energy range of 10-500 TeV and use off-source and off-time data to estimate background and to achieve blindness in the analysis.

Models of high energy neutrino emission from GRBs tend to focus on average properties, but several recent papers have asserted that rare, favorable fluctuations in the burst characteristics for one or two GRBs may dominate the neutrino flux. This suggests a two-fold search strategy: one designed to maximize the individual (or per-burst) sensitivity and another designed to maximize 
the composite sensitivity for all measured GRB. In this report, we focus on the simpler per-burst search strategy for the AMANDA-II analysis and note that the results from the composite search using the 1997 data from AMANDA-B10 were reported elsewhere(Hardke 2001).

The per-burst analysis of 2000 data from AMANDA-II looks for enhancements in the rate of upward-going muons over short periods from a fixed direction, with relatively loose background rejection requirements at the $10^{-4}$ level. Event rate stability over short periods is therefore an important measure of how effective this analysis can be. Figure 7 shows the count rate per $10 \mathrm{~s}$ bin in a time window of roughly \pm 1 hour around a particular GRB. The agreement with a Gaussian distribution shows that the detector did not experience instrumental effects which could mimic a GRB. Plots for all the other GRBs in the sample exhibit the same well-understood behavior.

All AMANDA-II data within the 2-hour window surrounding the GRB event were processed with full-iterative reconstruction of cleaned data. Cleaning procedures remove instrumental artifacts such as cross-talk signals. A very simple selection procedure achieved the requisite background rejection: the reconstructed declination of the AMANDA-II event had to be larger than -10 degrees and the angular direction had to be within 22 degrees of the GRB direction obtained from BATSE. The angular dependence of the background events and detector sensitivity is shown in Fig. 8. At the final cut level, we include the effect of neutrino absorption by the earth for the assumed spectrum with a spectral break at $500 \mathrm{TeV}$. Since the angular dependence for both signal and background is similar, the analysis does not benefit from the additional complication of angle dependent cuts. A total of 58 BATSE bursts were observed between February and May of 2000 (the sample includes triggered and non-triggered bursts to increase statistics). As shown in Fig. 9, the per-burst analysis reaches an effective area of $50,000 \mathrm{~m}^{2}$ for $E_{\nu}=100 \mathrm{TeV}$, approximately $50 \%$ of the effective area at trigger level. From Fig. 10 we conclude that search provides no evidence for correlated emission of high energy neutrinos from any GRB burst in the BATSE data sample from 2000. The histogram agrees with the line that was computed assuming random fluctuation of off-time background events.

In the AMANDA data spanning the years 1997-2000, we anticipate having a sample of roughly $500 \mathrm{GRBs}$ to search through. Once BATSE was decommissioned, the primary source of GRB alerts is the Interplanetary Network (IPN). The IPN database can be extended to include GRB with relatively poor localizations (Hurley 2002), given the large angular window employed by the AMANDA GRB analysis. The longer term prospects look promising as well. New satellites have either started operation in 2002 or planned for launch during the next half decade. They will enhance the GRB-finding capabilities of the GRB Coordinates Network (GCN 2002). Within the same time frame, the GRB sensitivity of AMANDA-II is planned to be augmented by the first strings of IceCube (Karle et al. 2002). As few as 16 additional strings can achieve an effective area of 0.5 $\mathrm{km}^{2}$ for neutrino energy in the theoretically interesting region around $100 \mathrm{TeV}$, although the potential of the configuration cannot match the full IceCube array. Figure 11 shows the volume-averaged effective area as a function of the muon energy at production. In this simulation, the strings are separated by $125 \mathrm{~m}$ and roughly centered on AMANDA-II. We point out that the configuration should 
achieve comparable detection area for generic searches for transient or episodic emission.

\section{LOOKING AHEAD}

As the analysis from AMANDA-II data in year 2000 is refined for publication, the focus will start to shift to data acquired during 2001 and 2002, with a commensurate factor of three increase in statistical precision. We are also currently analyzing the data from AMANDA-B10 acquired in 1998 and 1999. During the next two polar campaigns in Antarctica, the data acquisition system of AMANDA-II will be upgraded to handle much higher event rates and record the full signal waveforms from the optical modules. The added information will help to improve the energy response and angular resolution, especially for neutrinos with energies above $\mathrm{PeV}$. Then, as the analysis programs are modified to incorporate the new detector capabilities, AMANDA-II will reach its full potential. Beyond that, as few as 16 additional IceCube strings would dramatically boost the sensitivity of the system in several key physics goals and insure the continuation of state-of-the-art science output.

Acknowledgments. This research was supported by the following agencies: U.S. National Science Foundation, Office of Polar Programs; U.S. National Science Foundation, Physics Division; University of Wisconsin Alumni Research Foundation; U.S. Department of Energy; Swedish Research Council; Swedish Polar Research Secretariat; Knut and Alice Wallenberg Foundation, Sweden; German Ministry for Education and Research; U.S. National Energy Research Scientific Computing Center (supported by the Office of Energy Research of the U.S. Department of Energy); FNRS-FWO, Flanders Institute to encourage scientific and technological research in industry (IWT); Belgian Federal Office for Scientific, Technical and Cultural Affairs (OSTC), Belgium; UC-Irvine AENEAS Supercomputer Facility; Deutsche Forschungsgemeinschaft (DFG). D.F.C. acknowledges the support of the NSF CAREER program.

\section{References}

Ahrens, J. et al. 2001, Astropart. Phys., 16, 345

Ahrens, J. et al. 2002a, Phys. Rev. D, 012005

Ahrens, J. et al. 2002b, Phys. Rev. D, 032006.

Ahrens, J. et al. 2003a, ApJ, in press (astro-ph/0208006).

Ahrens, J. et al. 2003b, Phys. Rev. D, in press

Andres, E. et al. 2000, Astropart. Phys., 13, 1 (see www.ps.uci.edu/ amanda for more information)

Andres, E. et al. 2001, Nature, 410, 441

Balkanov, V. et al. 2001, in Proc. 9th Int. Workshop on Neutrino Telescopes (Venice, Italy) Vol. 3 p. 591

Barwick, S. W., 2000, Phys. Scripta, T, 85, 106 (astro-ph/9903467)

Bertin, V., Negri, E., \& Orloff, J. 2002, submitted to European Phy. J. C 

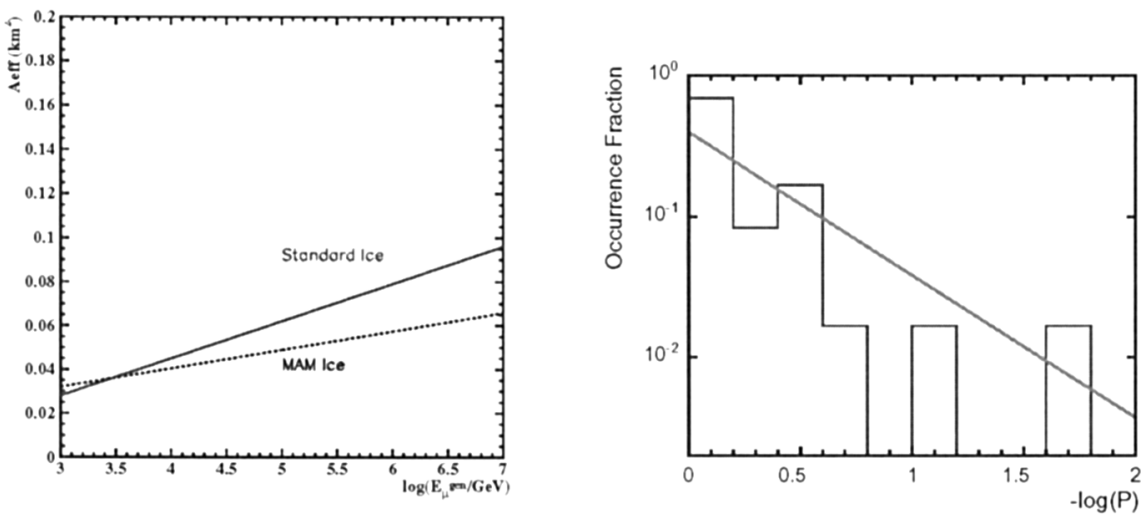

Figure 9. Left: The volume-averaged effective area as a function of the muon energy at production (typically a factor of 2 less than the incoming neutrino energy for charged current interactions). The effective area for the GRB analysis shown for two ice models and also averaged over declination. Statistical uncertainty is about $15 \%$ in this preliminary study.

Figure 10. Right: Histogram of chance probabilities (P) to obtain experimentally observed counts during the $\mathrm{T}_{90}$ window from a random fluctuation of expected background. The solid line was computed from off-time data of the GRBs by randomly redistributing the time stamps. The time-shuffling procedure was repeated 100 times and the results averaged.

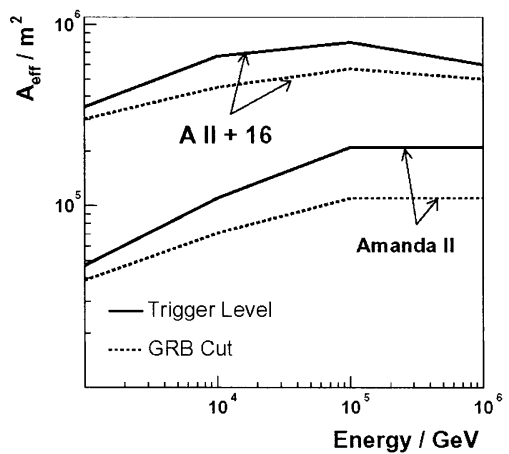

Figure 11. The volume-averaged effective area as a function of the muon energy at production for AMANDA-II and 16 additional strings. The effective area is averaged over declination. Solid lines correspond to hardware trigger with no background rejection. Dashed lines include background rejection to $10^{-5}$. Note that the trigger conditions for AMANDA-II in this study do not match the trigger conditions of the detector in 2000. 
Dermer, C. \& Atoyan, A. 2001, Phys. Rev. Lett. 87, 221102.

Gaisser, T. K., Halzen, F., \& Stanev, T., 1995, Phys. Rept. 258, 173

GCN, 2002, see http://gcn.gsfc.nasa.gov/

Gorham, P. et al. 2002, Proc. SPIE (Kona, Hawaii,)

Hardke, R. 2001, in Proc. 27th Int. Cosmic Ray Conf. (Hamburg, Germany)

Hill, G. C. \& Leuthold, M. J. 2001, in Proc. of ICRC, (Hamburg, Germany) p. 1113

Hurley, K., 2002, private communication.

Hundertmark, S., 2001, in Proc. Second Workshop Methodical Aspects Underwater/Underice Neutrino Telescopes, Hamburg (http://www.ps.uci.edu/ amanda/documents.html)

Karle, A. et al. 2002, Proc. XXth Int. Conf. Neutrino Phys. Astrophys. (Munich) (astro-ph/0209556)

Letessier-Selvon, A. et al. 2001, Proc. 27th ICRC, 1204

Levinson, A. \& Waxman, E., 2001, Phys. Rev. Lett., 871711101.01

Lipari, P., 1993, Astro. Part. Phys. 1, 193

Mannheim, K., Protheroe, R. J., \& Rachen, J. P., 2000, Phys. Rev. D, 63, 023003

Matsuno, S. 2001, Proc. Inter. Cosmic Ray Conf. (Hamburg, Germany) 1069

Niessen, P. \& Spiering, C., 2001, in Proc. of ICRC (Hamburg, Germany), p. 1496

Perrone et al. 2001, in Proc. 27th Int. Cosmic Ray Conf. (Hamburg, Germany), p. 1073

Price, P. B., Woschnagg, K., \& Chirkin, D., 2000, Geophys. Res. Lett., 27, 2129

Ringwald, A. \& Tu, H., 2002, Phys. Lett. B, 525, 135

Spiering, C. 2002, Prog. Part. Nucl. Phys., 48, 43

Waxman. E. \& Bahcall, J. 1997, Phys. Rev. Lett., 78, 2292

Waxman, E. \& Bahcall, J. 1999, Phys. Rev. D, 59, 023002 